\title{
Perfil da Demanda aos Cursos de Pós-Graduação, de 1996, na Área de Saúde Coletiva
}

\author{
Ana Cristina d'Andretta Tanaka ${ }^{1}$
}

\begin{abstract}
Resumo: Não se pode realizar a análise da Pós-Graduação em Saúde Coletiva sem o estudo da demanda. É necessário conhecer que tipo de profissional busca o curso para seu aperfeiçoamento profissional. Nesse sentido, o presente estudo analisou a totalidade dos profissionais que se candidataram à seleção nos 19 cursos de mestrado e nos 9 de doutorado existentes no país, para o ano de 1996. Para tanto, foram enviados a todos os cursos questionários para a coleta dos seguintes dados sobre a demanda: nome, idade, sexo, procedência, formação profissional, área de atuação e tipo de curso pretendido. De modo geral, os candidatos ao mestrado têm em média 35 anos, e provêm dos serviços de saúde (60\%), enquanto que os de doutorado têm em média 40 anos e estão atuando na docência e na pesquisa (70\%). Há uma maior procura para o mestrado do que para o doutorado, sendo que para ambos os cursos há uma demanda reprimida importante. A grande maioria dos candidatos é do sexo feminino (75\%), indicando a feminização do trabalho na área da Saúde Coletiva. Em relação à formação profissional há um forte predomínio da área biológica (70\%), seguido pelas ciências exatas e humanas. Em síntese, observou-se que o perfil da clientela do curso de mestrado é distinto do de doutorado. Em conseqüência disto há necessidade de planos curriculares específicos que atendam estas expectativas pessoais e profissionais, ampliando desta maneira a efetividade dos cursos, diminuindo as evasões e a insatisfação dos estudantes
\end{abstract}

Palavras-chave: Saúde Coletiva; Saúde Pública; Pós-Graduação; Análise da Demanda.

Summary: It is impossible to evaluate the Post-Graduation without a demand analysis. It is important to know what kind of professionals are seeking for Post-Graduation studies. The present research analyzes the profile of all candidates to the 19 master and 9 doctor degree courses in the field of Public Health, in Brazil, for the year 1996. A questionnaire was sent to each course asking for the following candidates data: name, age, gender, address, professional degree, working area and nature of the course pretended. In general, the master degree candidates are 35 years old and work in the health services (60\%), while the doctor degree candidates are 40 years old and work in teaching and research field (70\%). The master course has more candidates than the doctor degree courses, however both have an important unsatisfied demand. Most candidates are female (75\%), reflecting their predominance in the Public Health field. Biological sciences' graduates constitute the majority of candidates (70\%). Master course candidates profile is clearly distinct from the one of the doctor degree candidates. Therefore, it is necessary to have specific curricula adapted to their professional and personal expectancies in order to increase the courses effectiveness and to decrease the students dissatisfaction.

Keywords: Collective Health; Public Health; Post-graduation Courses; Demand Analysis.

1 Professora Associada (Livre-Docente) do Departamento de Saúde Materno-Infantil da Faculdacle de Saúde Pública da USP, SP. 


\section{Introdução}

Ao se proceder a um estudo criterioso sobre a Pós-Graduação na área de Saúde Coletiva, faz-se necessária uma análise da relação entre a oferta e a demanda.

A oferta, que é dada pela disponibilidade de cursos e vagas existentes no país vem, desde a reforma universitária ocorrida em 1968, apresentado um aumento crescente.

Em 1981, segundo Magaldi e Cordeiro (1984), havia, no país, 6 cursos de mestrado e 4 de doutorado; já em 1996 existiam 19 cursos de mestrado e 9 de doutorado. Este aumento não ocorreu homogeneamente no território nacional. Estes cursos se concentraram, predominantemente, na Região Sudeste onde encontram-se $47,4 \%$ dos cursos, sendo que nas demais Regiões a distribuição é a seguinte: $15,7 \%$, na Sul; $26,3 \%$, na Nordeste; $10,5 \%$, na Centro-Oeste. Na Região Norte não existe, até o momento, nenhum curso na área de Saúde Coletiva.

Esta distribuição é coerente com a concentração de escolas de nível superior brasileiras, que tem no eixo Sudeste e Sul o maior número de faculdades tanto públicas como privadas (MEC, 1996).

Os estudos sobre demanda à Pós-Graduação são praticamente inexistentes. Quem são estes alunos? De onde eles vêm? Qual a sua idade e formação? Por que buscam a área de Saúde Coletiva? Para onde eles vão? Estas questões não têm sido objeto de investigação.

Os estudos sobre demanda são tão escassos que, até em relação ao pós-graduado, isto é, ao egresso da Pós-Graduação, pouca atenção tem sido dada a ela. Para Bevilacqua et al. (1996): "raramente tem-se procurado fazer uma análise consistente da demanda por pessoal pós-graduado no Brasil. Talvez porque ela tenha sido pouco significativa até agora".

Porém, para se poder formular uma política coerente de formação de recursos humanos, faz-se necessário saber quem está sendo formado e qual é o mercado de trabalho a que ele se destina. Assim, a presente pesquisa tem por objetivo estudar o perfil do profissional que busca na Pós-Graduação em Saúde Coletiva o seu aperfeiçoamento profissional.

É importante ressaltar que esta pesquisa só se efetuou por apoio da ABRASCO, que, durante dois anos, não mediu esforços para que esta se realizasse. Parte desta investigação foi financiada pela CAPES.

\section{Metodologia}

Para se conhecer o perfil dos profissionais que procuram nos cursos de Pós-Graduação aperfeiçoamento profissional, foi enviado a todos os cursos um questionário sobre as características dos candidatos que concorreram à seleção para o ano de 1996.

Das 19 instituições sob estudo, 15 delas (78,9\%) enviaram informações, sendo que 14 com quase todos os dados e 1 (uma) muito parcialmente. Os 4 cursos que não responderam foram o Instituto Materno-Infantil de Pernambuco - IMIP, o NESC/CPqAM/ FIOCRUZ e dois cursos no Ceará. O Instituto Fernandes Figueira - IFF/FIOCRUZ somente informou sobre duas variáveis (gênero dos alunos e nível do curso - mestrado ou doutorado) o que obrigou a excluí-lo da análise, com exceção das variáveis referidas, consideradas, apenas, na apresentação geral dos dados. Em outras palavras, o presente trabatho refere-se a 14 cursos.

\section{Resultados e Discussão}

Para os cursos de 1996, concorreram à seleção 978 profissionais, sendo $730(74,6 \%)$ mulheres e 248 (25,4\%) homens. Em todos os cursos avaliados, esta relação foi sempre muito semelhante. Ao retirar para fins de análise a demanda de profissionais de 
áreas tradicionalmente femininas como enfermagem, nutrição e serviço social, a proporção de mulheres continuou sendo maior do que a de homens, com $68,6 \%$ e $31,2 \%$, respectivamente, reflexo da feminização do trabalho na área da Saúde Coletiva, visto ser esta menos rentável que outras especialidades.

Para Girardi, citado por Poz e Varella (1994), a participação feminina no trabalho em saúde cresceu da década de 70 para a de 80 de 40 para $60 \%$ do total da força de trabalho na saúde, repercutindo fortemente no nível de remuneração dos profissionais pois, historicamente, a mão-de-obra feminina é mais mal remunerada.

Em relação ao nível do curso, a grande maioria se inscreveu para o mestrado $86,1 \%$ (842), e apenas 13,9\% (136) para o doutorado.

A elevada porcentagem de profissionais que buscam aperfeiçoamento em nível de mestrado permite inferir que, no país, a PósGraduação stricto sensu constitui um recurso utilizado pelos profissionais para melhor se qualificar. Este fato será evidenciado de maneira mais clara quando as variáveis idade e área de atuação profissional forem analisadas, onde poder-se-á observar que os candidatos já são adultos com mais de 30 anos e, quase todos estão inseridos no mercado formal de trabalho.

Para evitar o viés de análise da demanda por nível, incluindo escolas que só possuem cursos de mestrado, optou-se por excluí-las. Assim, observou-se que em quase todas as instituições a procura, em média, foi cinco vezes maior para o mestrado do que para o doutorado. A Tabela 1 mostra bem este fato. Em 1996, os cursos ofereceram 279 vagas para o mestrado e 88 para o doutorado, totalizando 367 vagas. Ao cotejar o número de candidatos com o número de vagas disponíveis podese observar que em média havia 2,5 candidatos por vaga, dando lugar a uma demanda reprimida.
Ao analisar a demanda e a natureza do curso, observa-se que há maior procura pelo mestrado -3 candidatos por vaga -, do que para o doutorado - 1,5 candidato por vaga. É importante ressaltar que embora os dados indiquem que a demanda é maior para o mestrado do que para o doutorado, este fato talvez não revele a real magnitude do problema pois os candidatos ao doutorado em geral passam por uma seleção prévia à inscrição, isto é, procuram orientador antes de fazerem a inscrição para seleção; quando não o encontram disponível acabam não se inscrevendo, havendo, seguramente, uma demanda reprimida oculta.

Uma análise preliminar dos dados acerca das vagas permite inferir que a maioria dos cursos as definem de acordo com a disponibilidade de vagas dos seus orientadores credenciados, isto exige que o curso tenha um fluxo ágil de saída de alunos, pois, do contrário, o curso tenderá à estagnação.

Os dados, a partir deste ponto, serão analisados tendo como base os 907 profissionais dos 14 cursos com informações quase completas.

Pode-se observar, ainda na Tabela 1, a distribuição de candidatos por instituição. Como se nota, a grande maioria dos concorrentes é da Faculdade de Saúde Pública da USP, que é a primeira escola criada na área no país, seguida da Escola Nacional de Saúde Pública da FIOCRUZ e do Instituto de Medicina Social da UERJ, que são os maiores cursos do país nesta área, sendo também reflexo do grande número de vagas disponíveis e do grande número de orientadores que elas possuem.

As diferenças observadas entre as instituições estão talvez, primeiramente, relacionadas à disponibilidade de vagas e de orientação e, secundariamente, ao prestígio dos cursos.

Em relação à origem dos candidatos ${ }^{2}$, como era de se esperar, em sua maioria são eles da Região Sudeste, isto é, $44,5 \%$ são 
Tabela 1

Distribuição dos Candidatos a Pós-Graduação na Área de Saúde Coletiva de 1996, segundo Nivel e Instituição

\begin{tabular}{|c|c|c|c|c|}
\hline \multirow{2}{*}{ Instituição } & \multicolumn{2}{|c|}{ Mestrado } & \multicolumn{2}{|c|}{ Doutorado } \\
\hline & № & $\%$ & № & $\%$ \\
\hline Faculdade De Saúde Pública-FSP/USP & 239 & 85,1 & 42 & 14,9 \\
\hline Instituto De Medicina Social - IMS/UERJ & 140 & 88,6 & 18 & 11,4 \\
\hline Escola Nacional De Saúde Pública - ENSP/Fiocruz & 135 & 86,0 & 22 & 14,0 \\
\hline Instituto Fernandes Figueira - IFF/Fiocruz & 57 & 80,3 & 14 & 19,7 \\
\hline Pós-Graduação em Saúde Coletiva da UNICAMP & 46 & 73,0 & 17 & 27,0 \\
\hline Instituto De Saúde Coletiva-ISC/UFBa & 38 & 79,2 & 10 & 20,8 \\
\hline $\begin{array}{l}\text { Mestrado em Epidemiologia da Universidade Federal de Pelotas- } \\
\text { FMUFPel }\end{array}$ & 36 & 100,0 & não tem & não tem \\
\hline $\begin{array}{l}\text { Mestrado em Saúde Coletiva da Universidade Estadual de } \\
\text { Londrina - UEL }\end{array}$ & 31 & 100,0 & não tem & não tem \\
\hline $\begin{array}{l}\text { Pós-Graduação em Saúde Pública da Universidade Federal de Santa } \\
\text { Catarina-PGSP/UFSC }\end{array}$ & 30 & 100,0 & não tem & não tem \\
\hline $\begin{array}{l}\text { Mestrado em Saúde Coletiva da Universidade Federal do Mato } \\
\text { Grosso do Sul-UFMS }\end{array}$ & 28 & 100,0 & não tem & não tem \\
\hline $\begin{array}{l}\text { Mestrado em Epidemiologia da Universidade Federal de São Paulo - } \\
\text { EPMUNIFESP }\end{array}$ & 19 & 100,0 & não tem & não tem \\
\hline $\begin{array}{l}\text { Faculdade de Medicina da Universidade Federal de Minas Gerais - } \\
\text { FMUFMG }\end{array}$ & 19 & 100,0 & não tem & não tem \\
\hline $\begin{array}{l}\text { Instituto de Saúde Coletiva da Universidade Federal do Mato Grosso } \\
\text {-ISC/UFMT }\end{array}$ & 15 & 75,0 & 5 & 25,0 \\
\hline $\begin{array}{l}\text { Pós-Graduação em Medicina Preventiva da Faculdade de Medicina } \\
\text { daUSP-DMP/FM/USP }\end{array}$ & 4 & 44,4 & 5 & 55,6 \\
\hline $\begin{array}{l}\text { Pós-Graduação em Medicina Preventiva da Faculdade de Medicina } \\
\text { de Ribeirão Preto da USP - RP/USP }\end{array}$ & 5 & 37,5 & 3 & 62,5 \\
\hline Total & 842 & 85,9 & 136 & 14,1 \\
\hline
\end{tabular}

provenientes de São Paulo e 20,8\%, do Rio de Janeiro. A Tabela 2 mostra esta distribuição por Região e para o Estado de São Paulo e Rio de Janeiro; estes últimos por abarcarem mais de $50 \%$ dos profissionais.

É importante relatar que somente $0,9 \%$ dos candidatos eram da Região Norte ( 4 do Pará, 2 do Amazonas, um de Rondônia).

A Tabela acima mostra que os profissionais procuram basicamente os cursos de seus
Estados ou regiões. Embora haja nos cursos maiores candidatos de todas as Regiões do País, esta demanda é relativamente pequena, sugerindo que o profissional prefere não sair de seu ambiente de vida e trabalho para fazer seu aperfeiçoamento.

A análise da formação dos profissionais mostrou que há uma predominância de médicos (24,5\%) seguido dos psicólogos (14,3\%) e dos enfermeiros $(13,1 \%)$. A grande propor- 
Tabela 2

Distribuição Percentual dos Profissionais, por Região de Origem, que Demandaram aos Cursos de Pós-Graduação da Área de Saúde Coletiva de 1996

\begin{tabular}{l|ccccccc|c}
\hline \multirow{2}{*}{ Instituição } & \multicolumn{7}{c}{ Porcentagem } & No \\
\cline { 2 - 8 } & Norte & NE & SE & Sul & CO & SP & RJ & Total \\
\hline FSPNSP & 1,1 & 1,4 & 1,8 & 2,5 & 3,6 & 87,9 & 1,8 & 281 \\
IMSNERJ & - & 1,3 & 2,5 & - & 1,9 & 1,9 & 92,4 & 158 \\
UNICAMP & 3,2 & 1,6 & 3,2 & 4,8 & 3,2 & 79,4 & 4,8 & 63 \\
ISC/UFBA & 4,1 & 91,7 & - & - & 2,1 & - & 2,1 & 48 \\
FMUFPEL & - & - & - & 100,0 & - & - & - & 36 \\
UEL & - & - & - & 93,5 & 3,2 & 3,2 & - & 31 \\
PGSPNFSC & - & - & - & 100,0 & - & - & - & 30 \\
UFMS & - & - & - & - & 100,0 & - & - & 28 \\
ISCNFMT & - & - & - & - & 90,0 & 5,0 & 5,0 & 20 \\
EPMUNIFESP & - & - & - & - & - & 100,0 & - & 19 \\
FMUFMG & - & - & 94,7 & 5,3 & - & - & - & 19 \\
DMP/FMUSP & - & - & - & - & - & 100,0 & - & 9 \\
RPNSP & - & - & 50,0 & - & - & 50,0 & - & 8 \\
\hline Total & 1,0 & 6,8 & 4,4 & 14,1 & 8,4 & 44,5 & 20,8 & 750 \\
\hline
\end{tabular}

Tabela 3

Distribuição da Formação dos Profissionais que Demandaram a Pós-Graduação na Área de Saúde Coletiva em 1996, segundo Instituição

\begin{tabular}{|c|c|c|c|c|c|c|}
\hline \multirow[t]{2}{*}{ Instituição } & Medicina & Enfermagem & $\begin{array}{l}\text { Demais A. } \\
\text { de Saúde }\end{array}$ & C. Sociais & $\begin{array}{c}\text { Eng J } \\
\text { Arquit. }\end{array}$ & $\begin{array}{c}\text { Todas as } \\
\text { Demais }\end{array}$ \\
\hline & $\%$ & $\%$ & $\%$ & $\%$ & $\%$ & $\%$ \\
\hline FSPNSP & 16,0 & 13,2 & 44,1 & 7,0 & 7,1 & 12,7 \\
\hline ENSP/FIOCRUZ & 20,4 & 10,8 & 35,0 & 13,4 & 9,6 & 10,8 \\
\hline UNICAMP & 30,6 & 22,6 & 32,2 & 9,6 & 3,2 & 1,6 \\
\hline ISC/UFBa & 31,9 & 12,8 & 38,3 & 8,5 & 4,3 & 4,3 \\
\hline IMSNERJ & 20,3 & 7,6 & $55,0^{*}$ & 7,6 & 1,2 & 8,1 \\
\hline FMUFPel & 61,1 & 5,6 & 30,5 & 2,8 & - & - \\
\hline UEL & - & 41,8 & 38,8 & 6,5 & - & 12,9 \\
\hline PGSPNFSC & 26,7 & 40,0 & 23,3 & 3,3 & 3,3 & 3,3 \\
\hline UFMS & 7,1 & 10,7 & 57,1 & 14,3 & 3,6 & 7,1 \\
\hline ISCNFMT & 40,0 & 5,0 & 25,0 & 5,0 & 5,0 & 20,0 \\
\hline EPMUNIFESP & 57,9 & - & 26,4 & - & - & 15,7 \\
\hline FMUFMG & 47,4 & 5,3 & 47,4 & - & - & - \\
\hline DMP/FMUSP & 100,0 & - & - & - & - & - \\
\hline RPNSP & 100,0 & - & - & - & - & - \\
\hline Total & 24,5 & 13,1 & 41,0 & 7,8 & 4,9 & 8,7 \\
\hline
\end{tabular}

$43,7 \%$ de psicólogos. 
ção de psicólogos observada deveu-se ao curso do Instituto de Medicina Social da UERJ que, pela forma e campo de atuação, atrai estes profissionais. Em relação às outras áreas da saúde, há uma variedade muito grande de formação compreendendo desde veterinários, odontólogos até tecnólogos sanitaristas.

Nas outras áreas de formação - exatas e humanas - tem-se como demanda engenheiros, sociólogos, assistentes sociais, economistas, historiadores, entre outros.

A Tabela 3 resume a formação dos profissionais que buscam a Pós-Graduação na área de Saúde Coletiva mostrando, claramente, a multidisciplinaridade desta área.

Os cursos das duas Faculdades de Medicinas da USP (da capital e de Ribeirão Preto) só tềm candidatos de formação médica, por serem cursos destinados exclusivamente a estes profissionais. Os demais cursos possuem uma gama ampla de profissionais mostrando o caráter interdisciplinar destas instituições.

Em relação à idade dos profissionais, a análise desta variável mostrou que, em média, eles têm 35,2 anos, sendo de 34,5 anos para os que se inscreveram no mestrado e 40,3 anos para o doutorado. Esta média é considerada muito alta pelos organismos que fornecem bolsas de estudos no país. Mas, certamente, indica a realidade e a peculiaridade da Saúde Pública campo no qual, primeiramente, o profissional adquire experiência, buscando a Pós-Graduação somente depois de sentir necessidade de aprofundamento de seu conhecimento na área, muito diferente de outros campos da ciência em que o profissional necessita, cada vez mais, ter uma melhor qualificação para só depois conseguir entrar no mercado de trabalho.

O Gráfico 1 mostra a distribuição de candidatos por faixa etária, indicando que os candidatos ao mestrado se concentram entre 20 e 39 anos, enquanto os de doutorado, entre 30 e 54 anos.

A Tabela 4 mostra a média de idade dos candidatos por instituição e por nível. Nela, observa-se que, em quase todos os cursos, a média de idade para o mestrado situou-se ao redor dos 35 anos e para o doutorado, ao redor dos 40 anos.

Gráfico 1 - Distribuição dos Candidatos dos Cursos de Pós-Graduação da Área de Saúde Coletiva para 1996, por Faixa Etária

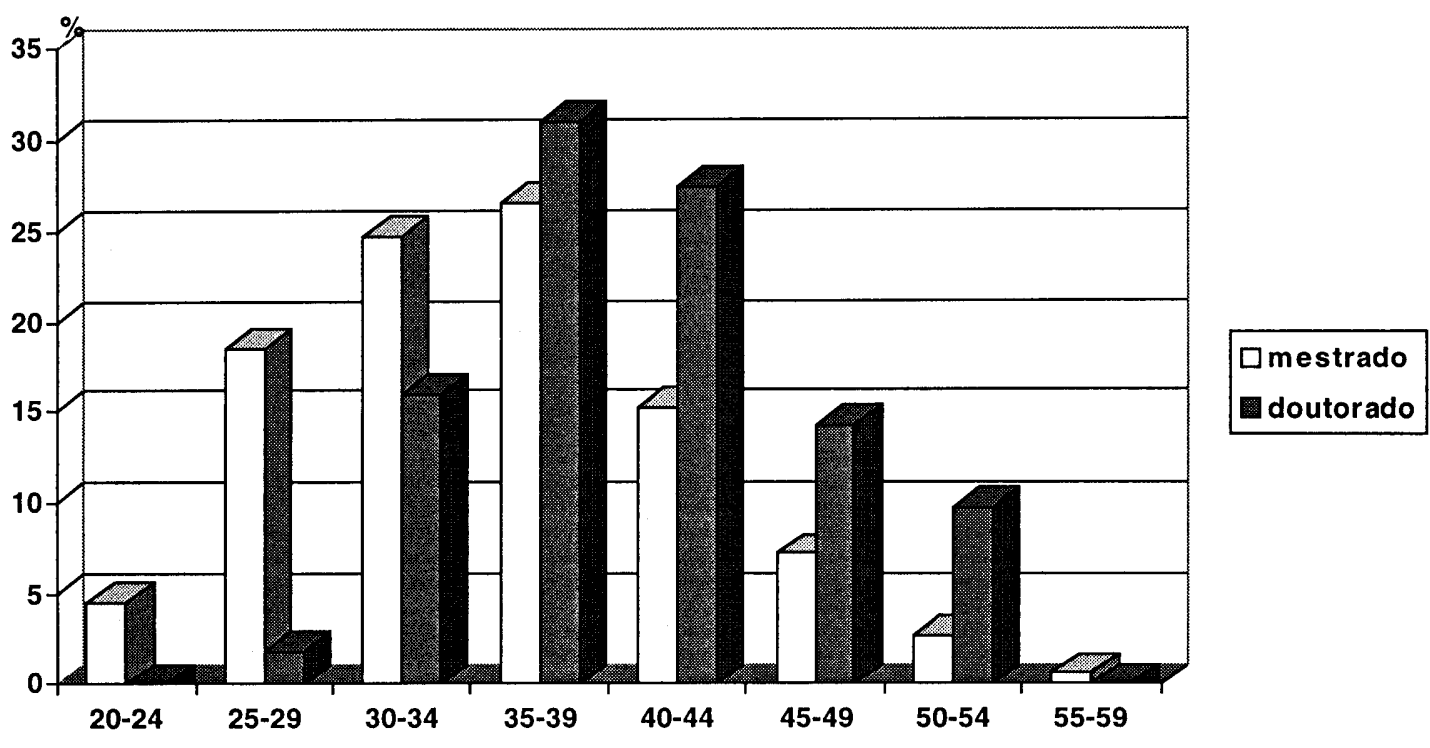


Tabela 4

Média de Idade dos Candidatos aos Cursos de Pós-Graduação da Área de Saúde Coletiva de 1996, segundo Instituição e Nível

\begin{tabular}{l|ccc}
\hline \multirow{2}{*}{ Instituição } & \multicolumn{3}{c}{ Média de Idade (Anos) } \\
\cline { 2 - 4 } FSPNSP & Geral & Mestrado & Doutorado \\
ENSP/FIOCRUZ & 35,2 & 34,4 & 41,1 \\
UNICAMP & 34,8 & 34,1 & 38,3 \\
ISC/UFBa & 35,7 & 34,8 & 38,8 \\
IMSNERJ & 35,4 & 34,0 & 40,7 \\
FMUFPel & 33,8 & 32,8 & 41,5 \\
UEL & 34,6 & 34,6 & - \\
PGSPNFSC & 34,8 & 34,8 & - \\
UFMS & $38,7^{\star}$ & 38,7 & - \\
ISCNFMT & 37,5 & 37,5 & - \\
EPMUNIFESP & 39,3 & 38,3 & 42,2 \\
FMUFMG & 37,7 & 37,7 & - \\
DMP/FMUSP & 34,5 & 34,5 & - \\
RPNSP & 41,9 & 41,3 & 42,4 \\
\hline Total & 40,0 & 37,4 & 44,3 \\
\hline * Dentre 30 candidatos, dispunha-se desta informação para apenas & 35,2 & 34,5 & 40,3 \\
\hline
\end{tabular}

Tabela 5

Distribuição dos Candidatos aos Cursos de Pós-Graduação na Área de Saúde Coletiva de 1996, segundo Área de Trabalho

\begin{tabular}{l|ccccc|c}
\hline \multirow{2}{*}{ Instituição } & Docência & Pesquisa & $\begin{array}{c}\text { Serv. } \\
\text { Saúde }\end{array}$ & $\begin{array}{c}\text { Fora da } \\
\text { Área }\end{array}$ & $\begin{array}{c}\text { Sem } \\
\text { Emprego }\end{array}$ & Total \\
\cline { 2 - 7 } & $\%$ & $\%$ & $\%$ & $\%$ & $\%$ & No \\
\hline FSPNSP & 14,5 & 9,2 & 67,1 & 1,7 & 7,5 & 173 \\
IMSNERJ & 54,4 & 11,4 & 26,6 & - & 7,6 & 79 \\
UNICAMP & 20,3 & 5,1 & 66,1 & - & 8,5 & 59 \\
ISC/UFBa & 30,2 & 7,0 & 51,2 & - & 11,6 & 43 \\
FMUFPel & 20,6 & 2,9 & 52,9 & - & 23,5 & 34 \\
UEL & 41,9 & - & 54,8 & - & 3,2 & 31 \\
PGSPNFSC & 26,6 & - & 70,0 & - & 3,3 & 30 \\
UFMS & 32,1 & - & 67,9 & - & - & 28 \\
ISCNFMT & 80,0 & 5,0 & 5,0 & 5,0 & 5,0 & 20 \\
EPMUNIFESP & 15,8 & 15,8 & 63,2 & - & 5,3 & 19 \\
FMUFMG & 21,1 & - & 63,2 & 5,3 & 10,5 & 19 \\
DMP/FMUSP & 66,7 & - & 33,3 & - & - & 9 \\
RPNSP & 25,0 & - & 62,5 & - & 12,5 & 8 \\
\hline Total & 29,2 & 6,5 & 55,4 & 0,9 & 8,0 & 552 \\
\hline
\end{tabular}


A diferença observada nos dois cursos das Faculdades de Medicina da USP, provavelmente, deve-se ao pequeno número de candidatos, e não a uma tendência destes cursos de contarem com alunos com mais idade.

Em relação ao tipo de trabalho dos profissionais, na Tabela 5, pode-se observar que $55,4 \%$ dos candidatos são de serviços públicos de saúde e que 29,2\% deles são da área de ensino superior. Esta Tabela mostra também que apenas $8,0 \%$ dos profissionais informaram não estar trabalhando. Para a análise desta variável não se contou com os dados da ENPS/FIOCRUZ, pois os candidatos não o informaram, sendo dela também excluídos os 198 casos $(26,4 \%)$ de "sem resposta" pertencentes às demais instituições.

A análise da área de atividade dos profissionais, segundo as instituições em que se inscreveram para seleção na Pós-Graduação, mostrou que apenas 7 cursos receberam candidatos da área de pesquisa e 3 de "Fora da Área", isto é, profissionais que trabalhavam em serviços públicos nas não propriamente em Saúde Pública, ou eram provenientes de serviços privados.

Apenas os cursos da Universidade Federal de Mato Grosso do Sul e da Faculdade de Medicina da USP (Capital) receberam demanda de profissionais que trabalhavam em docência ou em serviços públicos de saúde. Os demais contaram com candidatos de diversas áreas de atuação.
Ao relacionar a área de atuação do profissional segundo a média de idade dos mesmos, pode-se observar que os que atuavam na docência eram os mais velhos, seguidos dos servidores públicos da saúde, depois pelos pesquisadores e, finalmente, pelos de "fora da área e sem emprego" (Tabela 6).

Este resultado mostra como o docente, talvez por estar muito envolvido com as atividades acadêmicas, acaba tendo pouca disponibilidade de fazer Pós-Graduação e o faz muito mais tarde do que os outros que estão atuando em outras áreas.

Como era de se esperar, o profissional desempregado é mais jovem e, provavelmente, justamente por estar nesta condição, faz a Pós-Graduação como complementação de sua formação ou buscando este aperfeiçoamento para conseguir emprego.

A análise segundo nível do curso mostrou que o perfil dos profissionais que buscam o doutorado e o mestrado são bem diferentes. O Quadro 1 descreve estas diferenças.

Este Quadro ilustra bem as diferenças entre a demanda ao mestrado e ao doutorado. Enquanto entre os profissionais que demandam ao doutorado $59,3 \%$ são docentes e $10,4 \%$ pesquisadores, entre aqueles que procuram o mestrado a maioria é constituída de servidores públicos da área de saúde. É interessante notar também que a grande demanda se refere ao mestrado $(86,7 \%)$, e que os

Tabela 6

Média de Idade segundo Área de Trabalho dos Profissionais que se Candidataram aos Cursos de Pós-Graduação da Área de Saúde Coletiva de 1996

\begin{tabular}{l|cc}
\hline \multicolumn{1}{c|}{ Área de Trabalho } & Média de Idade (Anos) & NP \\
\hline Docência & 38,4 & 160 \\
Serviço de Saúde & 35,8 & 286 \\
Pesquisa & 33,8 & 36 \\
Fora da Área da Saúde Pública & 30,6 & 5 \\
Sem Emprego & 29,8 & 41 \\
\hline
\end{tabular}




\begin{tabular}{|c|c|c|c|}
\hline $\begin{array}{r}\text { Média de Idade e Áre } \\
\text { Selecionado } \\
\text { de Pós-Gra }\end{array}$ & $\begin{array}{l}\text { Quadro 1 } \\
\text { de Atuação, em Po } \\
\text { segundo Nível da } \\
\text { uação da Área de S }\end{array}$ & $\begin{array}{l}\text { ntagem, e } \\
\text { nanda aos } 0 \\
\text { le Coletiva, }\end{array}$ & $\begin{array}{l}\text { Candidatos } \\
\text { sos } \\
96\end{array}$ \\
\hline & & & \\
\hline & & Mestrado & Doutorado \\
\hline Média de Idade (anos) & & 34,5 & 40,3 \\
\hline Área de Atuação (\%) & - Docência & 24,2 & 59,3 \\
\hline & - Pesquisa & 5,9 & 10,4 \\
\hline & - Serviço de Saúde & 60,4 & 24,7 \\
\hline Selecionados (\%) & & 33,4 & 66,0 \\
\hline
\end{tabular}

candidatos ao doutorado são mais absorvidos que os primeiros.

Estas diferenças mostram o perfil destes profissionais por nível de curso, revelando que o candidato ao doutorado é, em geral, mais velho e está trabalhando, exercendo sua função na área do ensino superior e como pesquisador. Estes achados permitem inferir que o candidato ao doutorado busca na PósGraduação não só seu aprimoramento profissional mas também a ascensão na carreira universitária; visto ser este nível uma das exigências da carreira atual.

O candidato ao mestrado, por sua vez, é um pouco mais jovem, está trabalhando no serviço público de saúde e busca, provavelmente, na Pós-Graduação seu aperfeiçoamento profissional.

Desta forma, pode-se concluir que a demanda da Pós-Graduação na área de Saúde Coletiva tem duas clientelas diferentes, inseridas no mercado formal de trabalho, ambas buscando seu aperfeiçoamento, em local próximo, mas com objetivos seguramente distintos que deveriam ser vistos, pelos cursos, de acordo com suas reais necessidades e aspirações, de modo a evitar evasões, insatisfações e perda de investimentos, tanto por parte do órgão formador como das agências financiadoras.

\section{Referências bibliográficas}

BEVILACQUA, L. et al. (1996) - Formação de Pessoal

Pós-graduado e Pesquisa no Brasil. In: MEC (org.)

- Discussão da Pós-Graduação Brasileira,1996. Bra-

sília: Ministério da Educação e de Desporto.

MAGALDI, C. \& CORDEIRO, H. (1984) - Estado

Atual do Ensino e da Pesquisa em Saúde Coletiva no Brasil. In: Ensino da Saúde Pública, Medicina Preventiva e Social no Brasil. Rio de Janeiro: ABRASCO.
MEC (1996) - Desenvolvimento da Educação no Brasil. Brasília: Ministério da Educação e do Desporto.

POZ, M. R. D. \& VARELLA, T.C. (1994) - Recursos Humanos em Saúde no Brasil: Política e Problemas. In: Guimarães, R. \& Tavares, R. (orgs.) Saúde e Sociedade no Brasil - Anos 80. Rio de Janeiro: ABRASCO/Relume Dumará.

Agradecimentos: Agradecemos ao Sr. Nilson Silva Soares, funcionário do Departamento de Saúde Materno-Infantil da FSP/USP, pela coleta, elaboração do banco e digitação de dados sem os quais este trabalho não poderia ser realizado. 